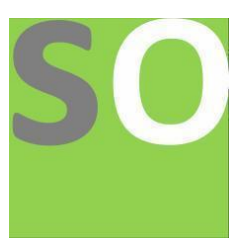

Article title: Natural phytochemicals, Phenformin, and Docosahexaenoic acid (DHA) as a Novel Inhibitors of IL-6 and ACE2 receptors, a Therapeutic Strategy for targeting COVID-19 Cell Entry and Cytokine Storm. An insilico Approach Authors: Amr Kamel Khalil Ahmed[1], Mahmoud Elkazzaz[2]

Affiliations: Director of tuberculosis program Ghubera, public health department ,First health cluster ,Ministry of health , Riyadh, Saudia Arabia.[1], Department of chemistry and biochemistry, Faculty of Science, Damietta University, Egypt[2]

Orcid ids: $0000-0003-3477-236 \times[1]$

Contact e-mail: drmedahmed@gmail.com

License information: This work has been published open access under Creative Commons Attribution License http://creativecommons.org/licenses/by/4.0/, which permits unrestricted use, distribution, and reproduction in any medium, provided the original work is properly cited. Conditions, terms of use and publishing policy can be found at https://www.scienceopen.com/.

Preprint statement: This article is a preprint and has not been peer-reviewed, under consideration and submitted to ScienceOpen Preprints for open peer review.

DOI: 10.14293/S2199-1006.1.SOR-.PPDSL3Y.v1

Preprint first posted online: 20 September 2021

Keywords: Ace2, Covid-19, phenoformin, molecular docking, bromealin, EGCG, DHA, interleukin 6, il-6 


\title{
Natural phytochemicals, Phenformin, and Docosahexaenoic acid (DHA) as a Novel Inhibitors of IL-6 and ACE2 receptors, a Therapeutic Strategy for targeting COVID-19 Cell Entry and Cytokine Storm. An insilico Approach
}

\author{
Amr Ahmed $^{1}$ and Mahmoud Elkazzaz ${ }^{2}$
}

1. Director of tuberculosis program Ghubera, public health department ,First health cluster ,Ministry of health, Riyadh, Saudia Arabia.

2. Department of chemistry and biochemistry, Faculty of Science, Damietta University, Egypt

3. Correspondence, Amr Ahmed. Email: drmedahmed@gmail.com

\begin{abstract}
Cytokine storm syndrome (CSS) is a life-threatening consequence of inflammatory immunological illnesses; it can also occur with COVID-19 infection. CSS is characterized by a disruption in cytokine synthesis, including regulatory, pro-inflammatory and anti-inflammatory cytokines, resulting in pathologic stimulation of innate in addition to adaptive (Th17 and Th1 mediated) response. In the pathophysiology of CSS, interleukin- 6 could play a key role. The significant role of IL-6 in COVID-19 pathogenesis was established in a wide variety of researches, which reported that the plasma concentration of IL- 6 was raised in COVID-19 patients with severe symptoms . COVID-19 spike protein binding to angiotensin-converting enzyme 2 (ACE2), the virus's cellular receptor, causes a cascade of molecular processes that could result in hyperinflammation which may lead to cytokine storm. Therefore, the development of new natural therapies and repurposing some drugs such as Phenformin and Docosahexaenoic acid that could compete with COVID-19 for ACE2 binding or inhibit IL-6 activity may possibly help COVID-19 patients avoid a cytokine storm and save their lives through inhibiting IL-6 and preventing SARS-CoV-2 RBD attachment to
\end{abstract}


ACE2. Herein we made a docking based screening for some natural phytochemicals and drugs that could be repurposed according to our findings to counter COVID-19 cell entry and inhibit the hyper activation of IL-6. Our results revealed that a five phytochemicals including Epigallocatechin gallate (EGCG), bromelain , luteolin , vitexin and isovitexin) showed a high binding affinities with best interactions with the active sites of IL- 6 . The binding affinities of these phytochemicals including, EGCG , bromelain, luteolin , vitexin and isovitexin with IL-6 were (-7.7, -6.7, -7.4, -7.2 and -7.3), respectively. In addition to, phenformin showed a high binding affinity with best interactions with the active sites of IL-6 and ACE2 . The binding affinity of phenformin with IL-6 was (-7.4) and with ACE 2 ( -7.2). Docosahexaenoic acid (DHA) had a moderate binding affinity and moderate interactions with the active sites of IL-6 and had a high binding affinity with best interactions with ACE2 active sites. The binding affinity of Docosahexaenoic acid(DHA) with IL-6 was (-5.3) and with AC2 (-6.3).

\section{Conclusion}

Proposing possible IL-6 inhibitors with less adverse effects has been suggested as a way to aid COVID-19 patients who are suffering from severe cytokine storms. This study has been designed to elucidate the potential of potent antiviral phytocompounds as well as phenformin and Docosahexaenoic acid (DHA) as a potent ACE2 and IL-6 inhibitors. The compounds interact with different active sites of IL6 and ACE2 which are involved in direct or indirect contacts with the ACE2 and IL-6 receptors which might act as potential blockers of functional ACE2 and IL-6 receptor complex. It worth mentioning that phenformin which showed high binding affinity with both ACE2 and IL-6 is currently under investigation for treating COVID-19 ClinicalTrials.gov Identifier: NCT05003492

Keywords: COVID-19, phytocompounds, phenformin and Docosahexaenoic acid,ACE2 and IL-6 


\section{Introduction}

The severe acute respiratory syndrome coronavirus 2 (SARS-CoV-2) an enveloped RNA virus that causes the recent pandemic of COVID-19 principally affects the human respiratory system but can also affect the nervous system, urogenital system, circulatory system and could also affect digestive system. ${ }^{1}$ COVID-19 belongs to family of Coronaviridae Because of the existing of crown-like spikes on its outer surface, the virus was given the name corona virus. ${ }^{2}$ Thousands of people have died as a result of infectious coronaviruses such as the Middle East respiratory syndrome (MERS) coronavirus and the severe acute respiratory syndrome (SARS) coronavirus in the last two decades.. ${ }^{3}$ The most recent outbreak of COVID-19 was discovered in late 2019 in Wuhan, China, where cases had pneumonia from an unknown etiology ... To combat this infectious virus, researchers must explore the pathogenicity mechanism of COVID-19 and how it acts with the immune system.. ${ }^{5}$ In COVID-19 patients who require ICU hospitalization, the cytokine storm may be present. It's a condition in which inflammatory cytokines are released in an uncontrolled manner. The molecular mechanism of the cytokine storm has yet to be fully elucidated. When the SARS-CoV-2 spike glycoprotein binds to the virus's cellular receptor, angiotensin-converting enzyme 2 (ACE2), a cascade of molecular events occurs, resulting in hyperinflammation ${ }^{6}$. COVID-19 infection has been linked to an increased response of the immune system in some covid-19 patients, which is governed by an excessive release of circulating cytokines known as cytokine release syndrome (CRS). ${ }^{7}$ One of the key causes of COVID-19 patients' significant 
deterioration, which leads to multiorgan failure, has been identified as cytokine release syndrome. ${ }^{8}$ Increased levels of interleukin (IL)-6, interferon (IFN)- , and tumour necrosis factor (TNF)-, are the most common features of a cytokine storm, which is known as a "cytokine storm." ${ }^{\text {IL-6 }}$ is a kind of interleukin that is produced by the Significant proinflammatory qualities play a critical role in ARDS, systemic inflammation, pneumonia associated with respiratory failure ${ }^{10}$ Interleukin-6 levels have been associated to the severity of COVID-19 infection and have been found to be high in individuals with respiratory dysfunction.. ${ }^{11}$ The quantity of interleukin-6 is linked to a higher risk of death, more than three times higher in individuals with complex COVID-19 than in those with simple disease..$^{12}$ As a result, IL-6 blocking drugs, as well as techniques aimed at decreasing this cytokine, have been successfully used in the treatment of persons with hyper-inflammatory conditions..$^{13}$ In addition, we suggest that medication that could compete with covid-19 for ACE2 may prevent COVID-19 entry and hyper inflammation and cytokine storm cascade. According to our findings many drugs such as, phenformin and active ingredient found in natural supplements like Docosahexaenoic acid (DHA) could be repurposed and screened for investigating their efficacy for competing with COVID-19 for ACE2 receptors a critical point that could prevent COVID-19 cell entry or inhibiting cytokine storm via binding directly to IL-6. Phenformin is a biguanide anti-diabetic drug that may be made in a single step chemical synthesis. It is an oral diabetes medication that aids in the regulation of blood sugar levels ${ }^{14}$.Phenformin has been shown to lower influenza mortality in mice. Because of the modest inhaled dose, buformin or phenformin inhalation for coronavirus could be a viable new treatment that reduces the danger of systemic side effects associated with biguanides ${ }^{15}$. As part of normal metabolism, certain algae create long chain omega-3 fatty acids such as eicosapentaenoic (EPA) and docosahexaenoic (DHA). These fatty acids are critical nutrients for the health of many species, including humans, when they enter the food chain in nature ${ }^{16}$.Docosahexaenoic acid (DHA) is an omega- $3(n-3)$ fatty acid with a long chain. It has a structure that provides it with distinct physical and functional characteristics ${ }^{17}$. Docosahexaenoic acid (DHA) is crucial for the 
growth and functional development of the brain in babies and is biologically connected to other n-3 fatty acids. DHA is also essential for adults to maintain optimal brain function ${ }^{17}$. According to our findings phenformin and Docosahexaenoic acid (DHA) may be used as a therapeutic agents against COVID-19 because of their inhibitory activity against IL-6 and ACE2 receptors of COVID-19.In addition, many natural components generated from plants, such as phytochemicals, may provide preliminary evidence for the use of plant extracts to suppress IL-6. Phytochemicals can provide a wide range of chemical diversity as well as anti-inflammatory properties, making them potentially useful as COVID-19 treatment agents.. Plants may be able to supply a number of low-cost medications that can standardize IL-6 levels.. ${ }^{18}$ The use of phytochemicals as anti-IL- 6 agents could thus be a helpful technique for decreasing COVID-19's negative effects. ${ }^{19}$

In this study, EGCG, bromelain, luteolin, vitexin and isovitexin are found to be potential phytocompounds to inhibit the IL- 6 along with phenformin and Docosahexaenoic acid (DHA) .The Targeted 3-dimensional (3D) protein structures were obtained from the Protein Data Bank (PDB). Antiviral chemicals were chosen from a list of antidiabetic drugs and plantbased phytocompounds gathered from the literature. The aim of the in silico study is to identify the efficacy of (EGCG, bromelain, luteolin, vitexin and isovitexin) production along with phenformin and Docosahexaenoic acid (DHA) that can be tested as potential candidates against COVID-19.

\section{Materials and Methods}

\section{Sequence retrieval}

Structure of IL-6 and sequence were obtained from Protein Data Bank PDB under Protein Data Bank PDB iD (1alu), https://www.rcsb.org/structure/1ALU 


\section{Selection of drugs that could be repurposed against IL-6 and ACE2}

After extensive literature these drugs (phenformin and Docosahexaenoic acid (DHA) ) were selected based on their antiviral power. ADMET (Excretion, Absorption, Metabolism , Transport, and Toxicity) properties were calculated by admetSAR server(admetSAR was developed as a comprehensive source and free tool for the prediction of chemical ADMET properties. )..$^{20}$

\section{Selection of Phytocompounds that could have inhibitory activity against IL-6 and ACE2}

After extensive literature, 13 phytocompounds (quercetin, Epigallocatechin gallate (EGCG), wedelolactone ,bromelain, catechin, luteolin , nimbin , vitexin and eucalyptol, isovitexin, azadirachtin, kaempferol ad melanoxetin) were selected based on their antiviral activity. The 2D structures of selected phytocompounds were drawn by ChemDraw and retrieved as PDB format. ${ }^{21}$ UCSF Chimera 1.12 was used to do energy minimization on all identified phytocompounds. The top three phytocompounds were also chosen based on binding affinities $(\mathrm{Kcal} / \mathrm{mol})$. Using the mCule server, the Lipinski's rule of 5 was determined. The admetSAR server estimated 21 ADMET (Absorption, Distribution, Metabolism, Excretion, and Toxicity) characteristics. ${ }^{20}$

\section{Interaction studies}

The protein-ligand interactions and binding conformational behaviour within the active pocket of the targeted protein IL 6 were studied using molecular docking.. The active sites of IL6 were found in the literature prior to online tool using Galaxy binding site server ad docking trials. ${ }^{22}$. $^{23}$ The active site residues of a protein were surrounded by a grid. using HDOCK server was used to perform docking experiment. ${ }^{24}$ 


\section{Molecular docking of proteins and ligands}

The binding mode of Spike -ACE 2 and ligands of Docosahexaenoic $\operatorname{acid}(\mathrm{DHA})$ and phenformin which its retrieved form the PDB https://www.rcsb.org/ with accession number (7DMU , 5JOZ and 5UIH) representatively . the Spike -ACE 2 and ligands of both Docosahexaenoic $\operatorname{acid}(\mathrm{DHA})$ and phenformin were investigated to determine the conservative residues of binding of Spike protein with the ACE to be a control results also, ACE2 with ligands of Docosahexaenoic acid(DHA) and phenformin to know and discover if the ACE2 receptor of the virus are bind with ligands of Docosahexaenoic acid(DHA) and phenformin in a good binding affinity to declare the mechanism of the interaction. protein .Docking study of each Spike -ACE 2 and ligands of Docosahexaenoic acid(DHA) and phenformin were carried out using HDOCK server There are two working modes in the server :one is the default hybrid docking mode, and the other is the templatefree docking mode. First, we put the spike protein after we download it from pdb in accession 6MOJ we enter it on SAMSON software to separate the ACE2 and Spike protein to make docking between them to get the result as a control result to test and know how the complex of spike ligands of Docosahexaenoic acid(DHA) and phenformin are how much its efficient and submit into the server, one for ligands of Docosahexaenoic $\operatorname{acid}(\mathrm{DHA})$ and phenformin and the other for ligand (ACE2), in which both amino acid sequences and PDB structures are supported. Then, the server do the template-based modeling of the receptor and ligand molecules by searching the PDB for putative homologous templates based on the sequences of proteins. Then it's found the PDB ID of the two protein then the HDOCK server perform global docking to sample putative binding modes through an FFT-based search method and then evaluate them without intrinsic scoring function for protein-ligand interactions. Biological information, such as experimental data on the protein-ligand binding site or SAXS profile, can be incorporated during the docking and/or post-docking processes. To offer data on docking energy scores, The ligand RMSDs from the input structures or modelled structures of the interface residues within 5.0 of their interacting partner or each other, to get the corresponding distances about the residue contacts between proteins and ligands to aid in evolutionary analysis in sequences 17 or deep learning18. Finally, users can download the top 100 
predicted complex structures, with the top 10 models shown using an interactive NGL viewer36 on the result web page.

\section{Binding free energy calculation}

The MMGB/PBSA approach was used to calculate the binding affinities of the optimal docking conformations acquired as a result of molecular docking. 25 The computations were carried out using the AMBER MMPBSA.py model. ${ }^{25}$ The following formulas were used to determine binding free energy using Prime v3.5 and four protein-inhibitor complexes and their binding modes:

\section{1- $\Delta$ Gbind=Gcomplex $-($ Gprotein+Gligand $)$}

2- $\Delta$ Gbind $=\Delta H-T \Delta S=\Delta E M M+\Delta G s o l-T S$

3- $\Delta E M M=E i n t e r n a l+E v d w+E e l e$

4- $\Delta$ Gsol $=\Delta G P B / G B+\Delta G S A$

The gas-phase MM energy, the solvation free energy, and the conformational entropy are represented by E MM, G sol, and TS, respectively. In EMM, all bond, angle, and dihedral energies were addressed, as well as electrostatic internal energies (Eele) and the van der Waal's (Evdw). The polar contribution was calculated using the GB or PB models, while $G$ was the sum of electrostatic solvation energies.

\section{Results and discussion}

\section{Interaction analysis}

The best computational approach for exploring the active sites of proteins and the conformational position of ligands within the active pocket of a specific protein is molecular docking. . The binding affinities (Kcal/mol), 
molecular interactions, and bonding interactions of the docked complexes formed by molecular docking studies were investigated. The ligand's optimal structural position within the active region of the targeted protein ACE2 and IL-6 is represented by the lowest binding energy value. The docking results showed that the Five phytochemicals including EGCG, bromelain, luteolin, vitexin and isovitexin) show high binding affinity with best interactions with the active site of IL-6 . The binding affinities of the Five phytochemicals including EGCG, bromelain, luteolin, vitexin and isovitexin) are (-7.7, -6.7, -7.4, -7.2 and -7.3) respectively. In addition, phenformin shows high binding affinity with best interactions with the active sites of IL-6 and ACE2 . The binding affinity of phenformin with IL-6 was (-7.4) and with ACE 2 was( -7.2). Docosahexaenoic acid(DHA) shows moderate binding affinity with moderate interactions with the active sites of IL-6 and ACE2. The binding affinity of Docosahexaenoic acid(DHA) with IL-6 was (-5.3) and with AC2 (-6.3) The binding affinities of each compound with IL-6 and ACE2 are listed in Table 1, Table2 and Table 3

Table1: The binding affinities between phytochemicals and II-6

\begin{tabular}{|l|l|}
\hline Phytochemicals & Binding Affinity with IL-6 \\
\hline Bromelain & -6.7 \\
\hline EGCG & -7.7 \\
\hline isovitexin & -7.3 \\
\hline Luteolin & -7.4 \\
\hline Vitexin & -7.2 \\
\hline
\end{tabular}

Table2 : The binding affinities of II-6 with Phenformin and Docosahexaenoic acid (DHA)

Phenformin and Docosahexaenoic acid (DHA) Binding Affinity with IL-6 Binding Affinity with ACE2 


\begin{tabular}{|l|l|l|}
\hline Phenformin & -7.4 & -7.2 \\
\hline Docosahexaenoic acid (DHA) & -5.3 & -6.3 \\
\hline
\end{tabular}

\section{Active bonds with IL-6}

The interactive residues of each compound with IL-6 are showed in (Fig 1). Docking of bromelain with interleukin 6, the two-dimensional interaction between Bromelain and residues of IL-6. Most important hydrogen bonds were made by GLU_A:43, LYS_A:47 (It also made some hydrophobic interactions with Bromelain), ARG_A:105, GLN_A:157, and GLN_A:160. GLU_A:107 and THR_A:164 show unfavorable $H$-Bonds which are quite acceptable for rigid docking. Docking of Docking of EGCG with interleukin 6, the two-dimensional interaction between EGCG and residues of IL- 6 showed that the most important hydrogen bonds were made by ASP A:161, (It also made some hydrophobic interactions with EGCG), GLU_A:43 as showed in fig2. Docking of isovitexin with interleukin 6, showed the two-dimensional interaction between isovitexin and residues of IL-6. Most important hydrogen bonds were made by GLU_A:60, ASN_A:61 (It also made some hydrophobic interactions with isovitexin), LYS _A:67, GLU_A 60 , and ARG_A:169. GLU_A:107 and THR_A:164 show unfavorable H-Bonds which are quite acceptable for rigid docking as showed in fig3 . Docking of luteolin with interleukin 6, the two-dimensional interaction between luteolin and residues of IL-6. Showed that the most important hydrogen bonds were made by SER_A:108, (It also made some hydrophobic interactions with luteolin), ASP_A:161, ARG_A:105, and GLN_A:157. and THR_A:44 as showed in fig4.. Docking of vitexin with interleukin 6, the two-dimensional interaction between vitexin and residues of IL-6 showed that the most important hydrogen bonds were made by ARG_A:105, GLU A: 43,THR A:164 and GLN A: 160 as showed in fig5. Docking of Phenformin with interleukin 6, the two-dimensional interaction between Phenformin and residues of IL-6 showed that the most important hydrogen bonds were made by GLN A:160 , ASP A:161 (It also made some hydrophobic interactions with Phenformin), ASP A:161 and PHE A : 106.as showed in fig6. Docking of docking of DHA with interleukin 6, the two-dimensional 
interaction between DHA and residues of IL-6. Most important hydrogen bonds were made by THR_A:164 (It also made some hydrophobic interactions with DHA), THR_A:44 as showed in fig8.

\section{Active bonds with ACE2}

\section{Phenformin with ACE2}

Docking of Phenformin with interleukin 6, the two-dimensional interaction between Phenformin and residues of ACE2 showed that the most important three hydrogen bonds were made by GLU_A:208 (It also made some hydrophobic interactions with Phenformin),VAL A:212, LEU_A:95, and PRO A:565 as showed in fig 7 .

\section{DHA with ACE2}

Docking of DHA with ACE2, the two-dimensional interaction between DHA and residues of ACE2 showed that the most important three hydrogen bonds were made by SER_A:44 and SER A: 47 (It also made some hydrophobic interactions with DHA),PHE A:390, , and PHE A:40, as showed in fig 9 The compounds interact with different sites of IL6 and ACE2 which are involved in direct or indirect contacts with the ACE2 and IL-6 receptor which might act as potential blockers of functional ACE2 and IL-6 receptor complex. 

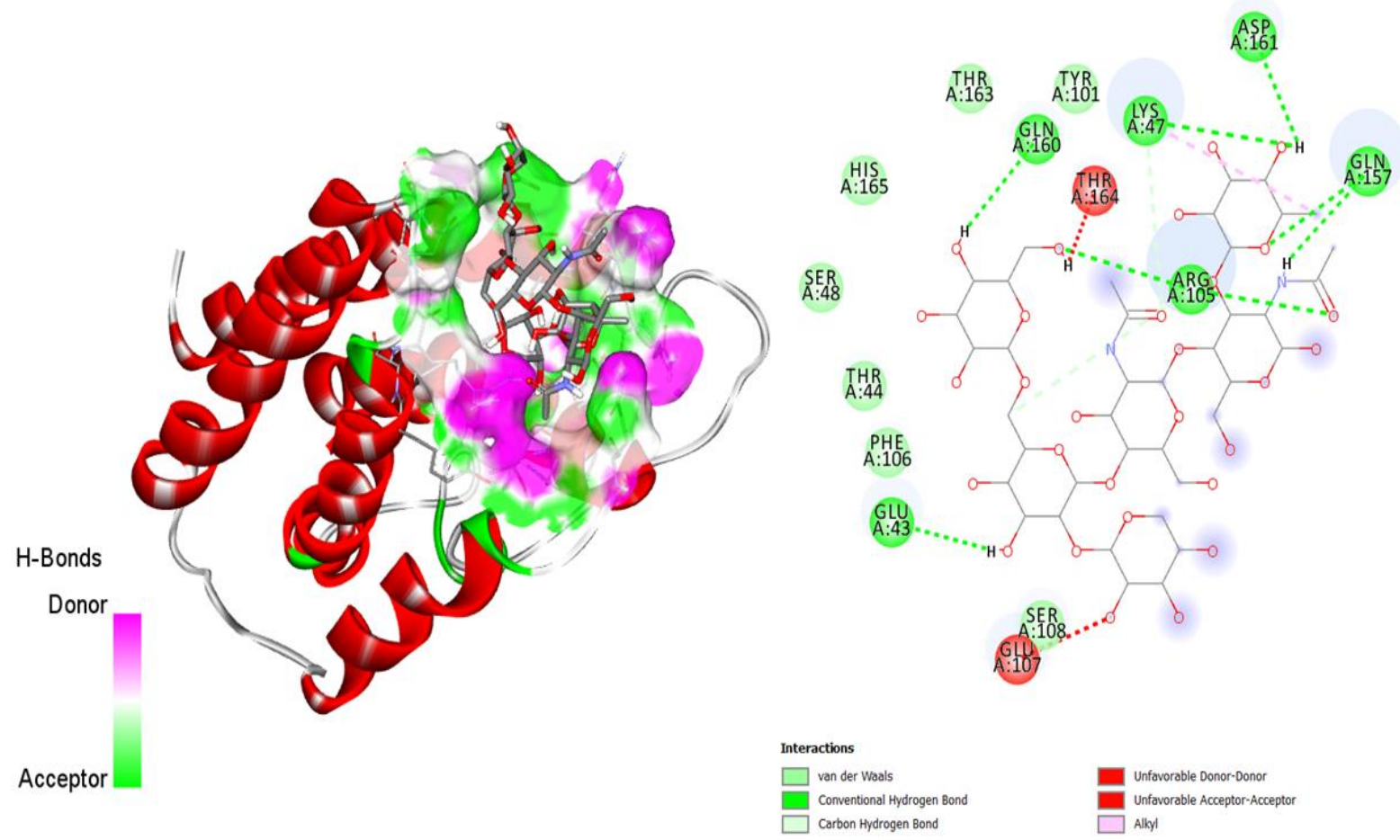

Figure 1: " shows docking of bromelain with interleukin 6, the twodimensional interaction between Bromelain and residues of IL-6. Most important hydrogen bonds were made by GLU_A:43, LYS_A:47 (It also made some hydrophobic interactions with Bromelain), ARG_A:105, GLN_A:157, and GLN_A:160. GLU_A:107 and THR_A:164 show unfavorable H-Bonds which are quite acceptable for rigid docking."
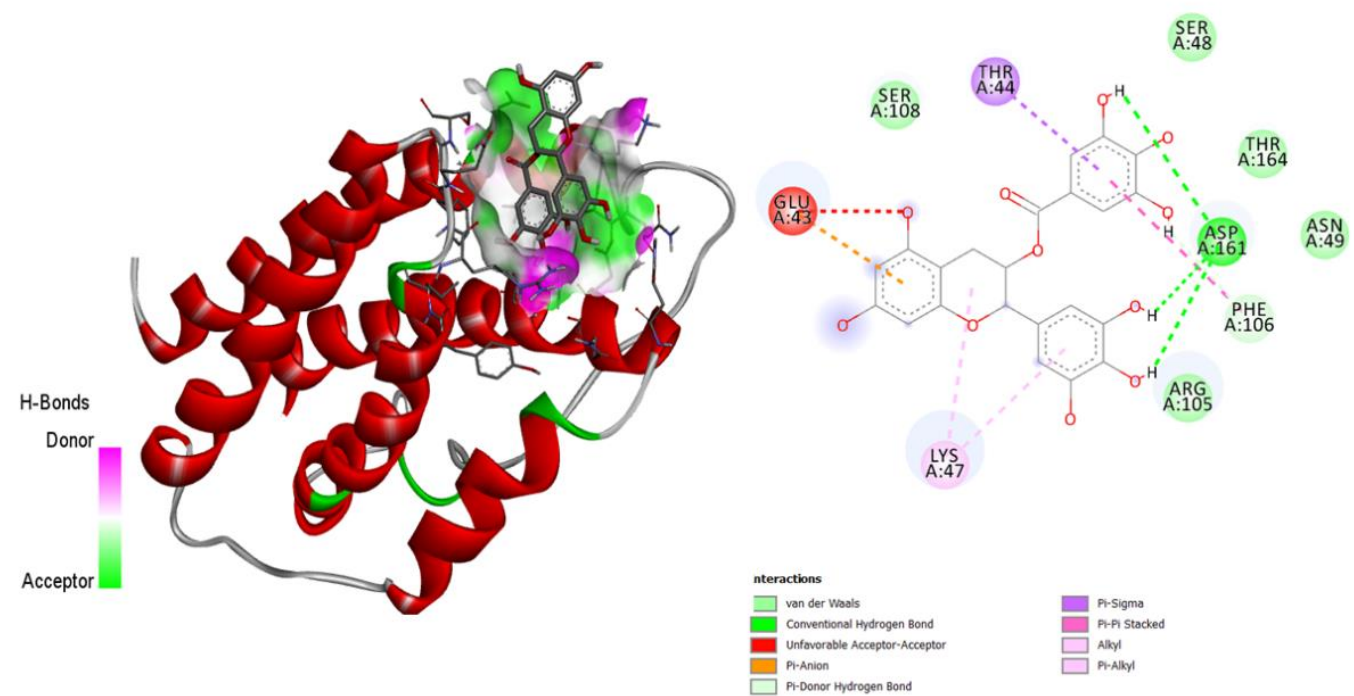

Figure2: shows docking of EGCG with interleukin 6, the two-dimensional interaction between EGCG and residues of IL-6. Most important hydrogen bonds 
were made by ASP A:161, (It also made some hydrophobic interactions with EGCG), GLU_A:43
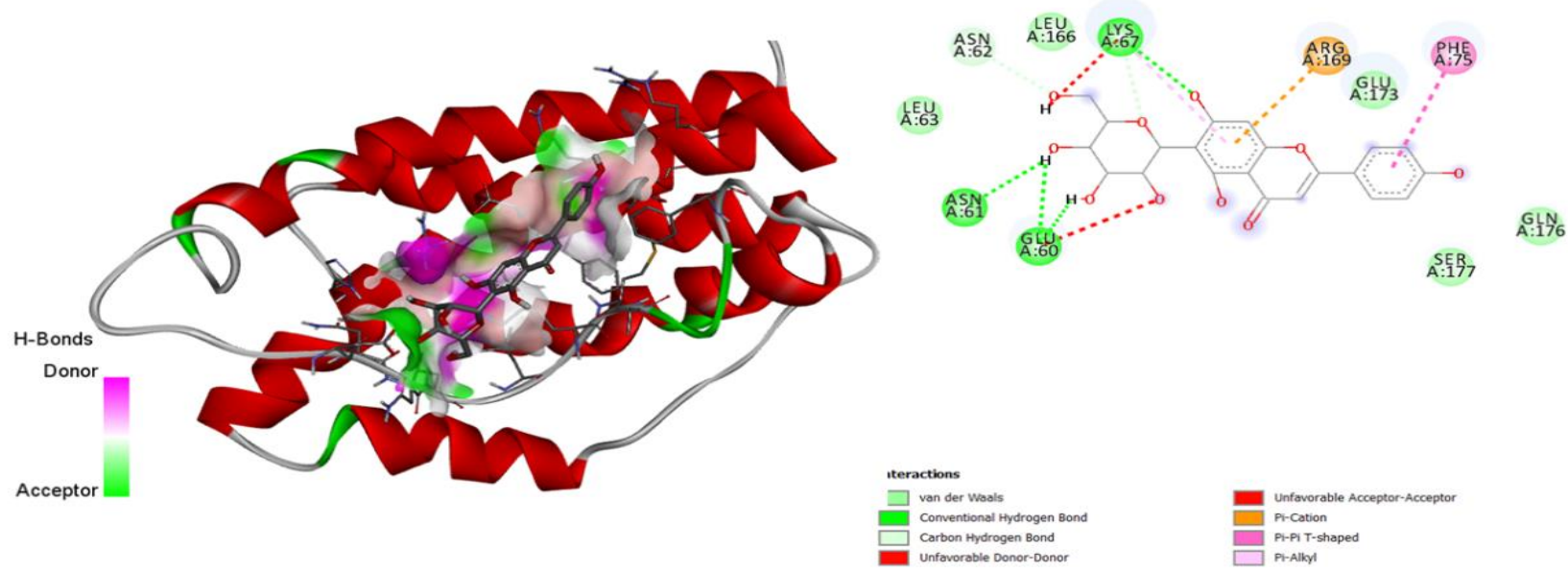

Figure 3: " shows docking of isovitexin with interleukin 6, the two-dimensional interaction between isovitexin and residues of IL-6. Most important hydrogen bonds were made by GLU_A:60, ASN_A:61 (It also made some hydrophobic interactions with isovitexin), LYS_A:67, GLU_A 60 , and ARG_A:169. GLU_A:107 and THR_A:164 show unfavorable H-Bonds which are quite acceptable for rigid docking."
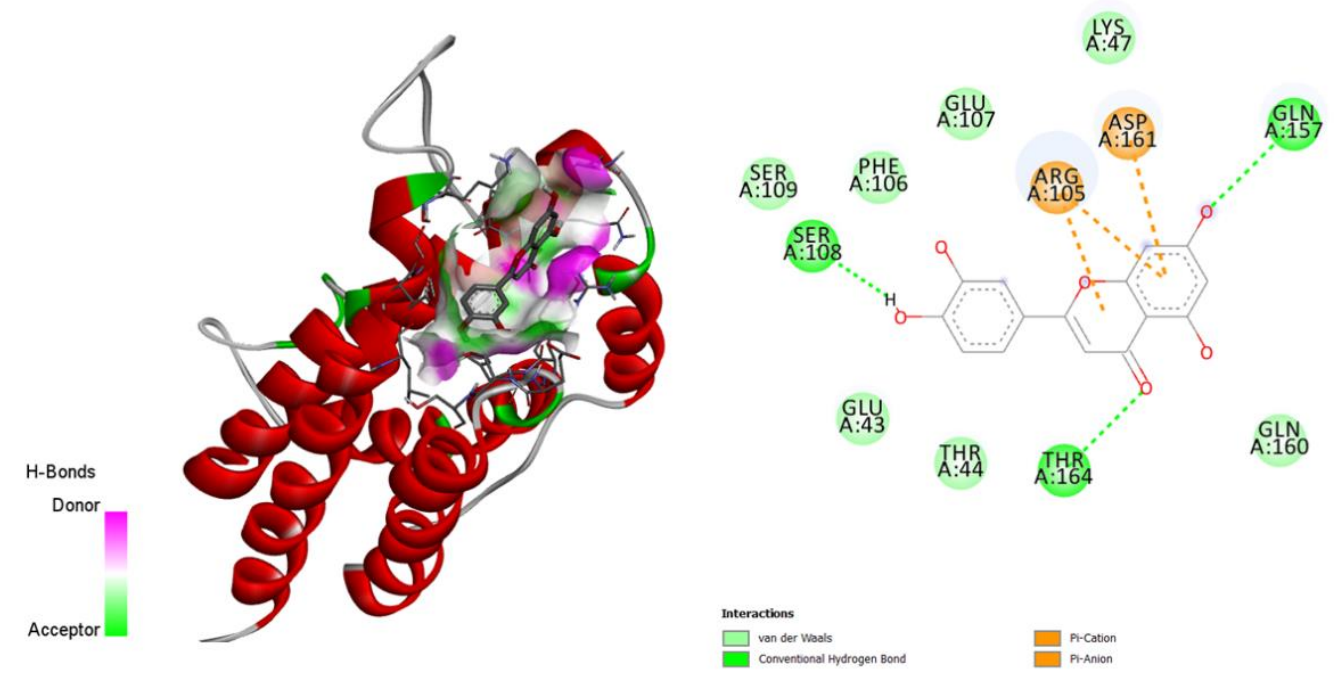

Figure 4: " shows docking of luteolin with interleukin 6, the two-dimensional interaction between luteolin and residues of IL-6. Most important hydrogen 
bonds were made by SER_A:108, (It also made some hydrophobic interactions with luteolin), ASP _A:161, ARG_A:105, and GLN_A:157. and
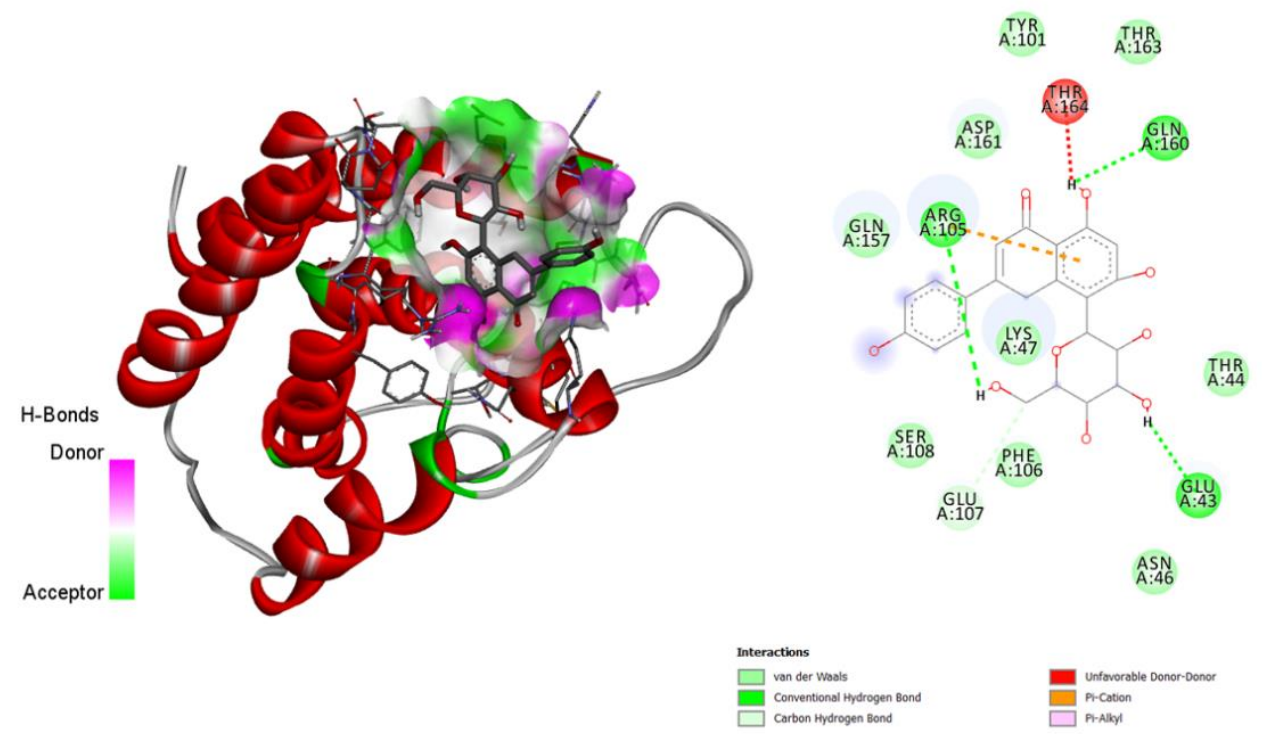

Figure 5: shows docking of vitexin with interleukin 6, the two-dimensional interaction between vitexin and residues of IL-6. Most important hydrogen bonds were made by ARG_A:105, GLU A: 43,THR A:164 and GLN A: 160 


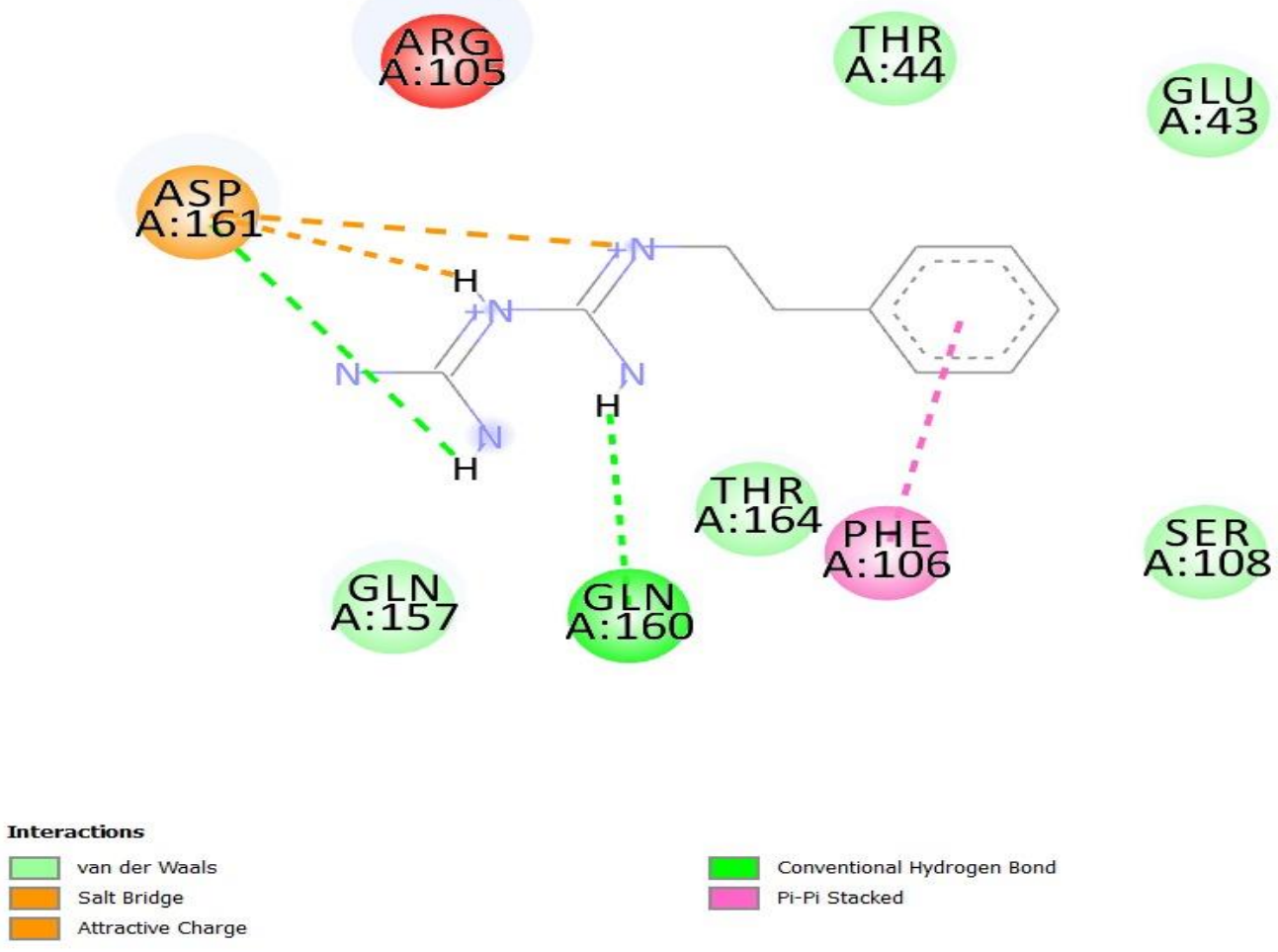

Figure 6: " shows docking of Phenformin with interleukin 6, the twodimensional interaction between Phenformin and residues of IL-6. Most important hydrogen bonds were made by GLN A:160, ASP A:161 (It also made some hydrophobic interactions with Phenformin), ASP A:161 and PHE A : 106. 


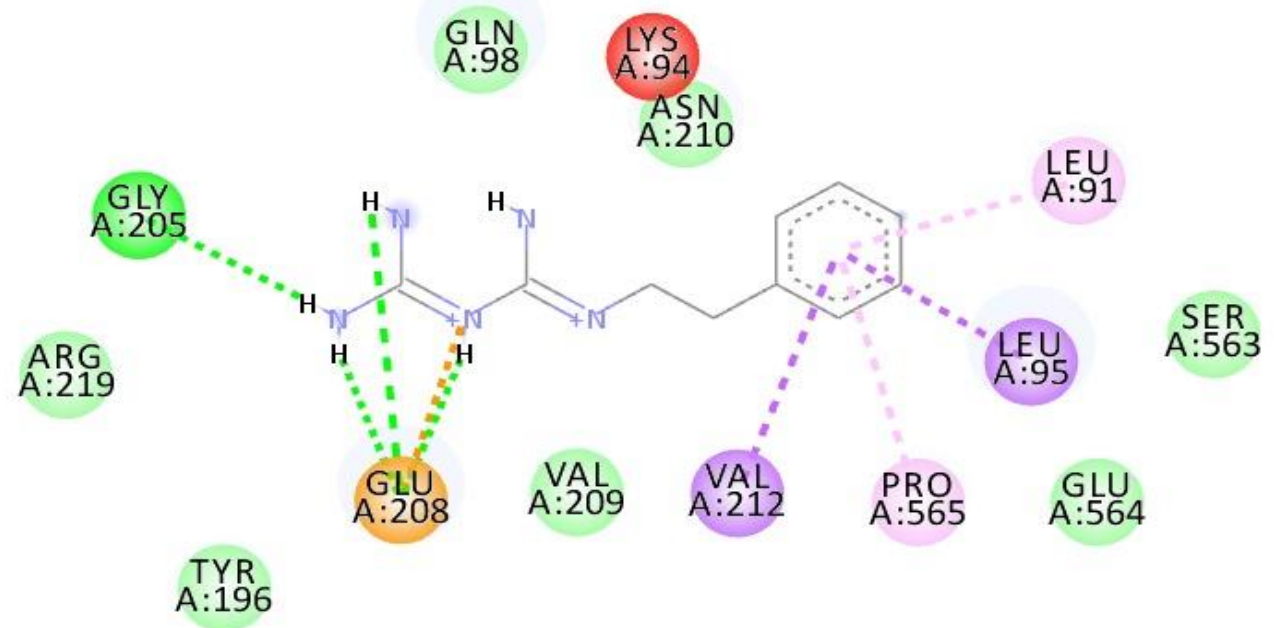

Interactions

$\square$ van der Waals
Attractive Charge

Figure 7: " shows docking of Phenformin with ACE2, the two-dimensional interaction between Phenformin and residues of ACE2. Most important three hydrogen bonds were made by GLU_A:208 (It also made some hydrophobic interactions with Phenformin),VAL A:212, LEU_A:95, and PRO_A:565. 


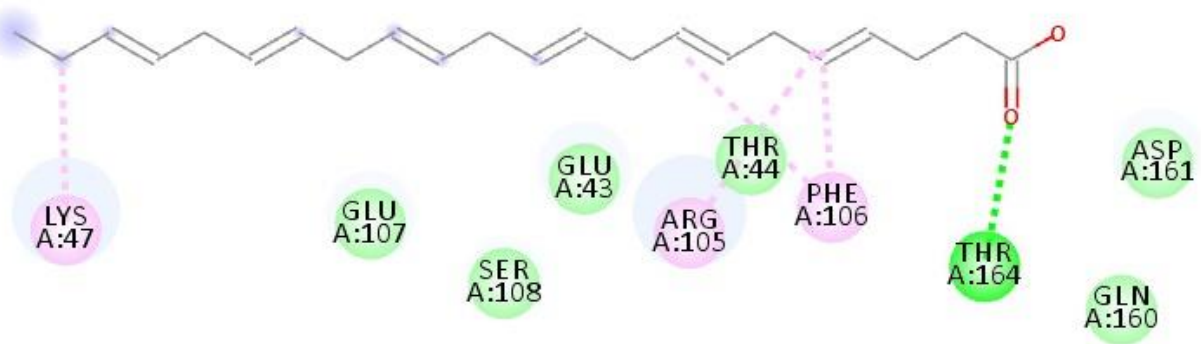

Interactions

$\square$ van der Waals Alkyl

Conventional Hydrogen Bond Pi-Alkyl

Figure 8: " shows docking of docking of DHA with interleukin 6, the twodimensional interaction between DHA and residues of IL-6. Most important hydrogen bonds were made by THR_A:164 (It also made some hydrophobic interactions with DHA), THR_A:44 


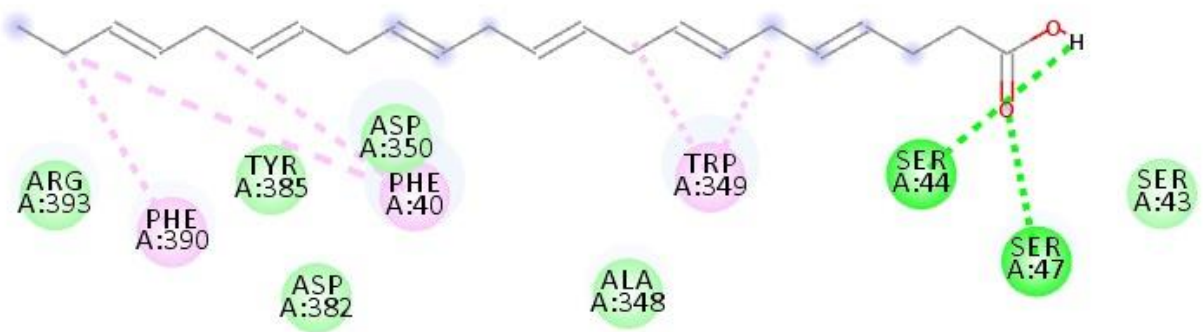

Interactions

van der Waals

Conventional Hydrogen Bond

Figure 9: " shows docking of DHA with ACE2, the two-dimensional interaction between DHA and residues of ACE2 showed that the most important three hydrogen bonds were made by SER_A:44 and SER A: 47 (It also made some hydrophobic interactions with DHA),PHE A:390, , and PHE A:40. 


\section{Conclusion}

Our findings demonstrated the possible efficacy of the investigated compounds including Epigallocatechin gallate (EGCG), bromelain, luteolin, vitexin and isovitexin as well as phenformin and Docosahexaenoic acid (DHA) as a potent inhibitor of Il-6 and ACE 2. Therefore, theses compounds could be used as a novel treatments for inhibiting COVID-19 cell entry and preventing its inflammatory complication

\section{Conflict of Interest Statement}

The author declares that the research was conducted in the absence of any commercial or financial relationships that could be construed as a potential conflict of interest

\section{References}

1-Cui, J, Li, F, Shi, Z-L. Origin and evolution of pathogenic coronaviruses. .Nat Rev Microbiol. 2019;17:181-192

2- Zhong, N, Zheng, BJ, Li, YM, et al. Epidemiology and cause of severe acute respiratory syndrome (SARS) in Guangdong, People's Republic of 'China, in February, 2003. Lancet. 2003;362:1353-1358.

3- Yang, $\mathrm{X}, \mathrm{Yu}, \mathrm{Y}, \mathrm{Xu}, \mathrm{J}$, et al. Clinical course and outcomes of critically ill patients with SARS-CoV-2 pneumonia in Wuhan, China: a singleicentered, retrospective, observational study. Lancet Respir Med. 2020;8:475-481.

4- Epidemiology Working Group for NCIP Epidemic Response, Chinese 'Center for Disease Control and Prevention. The epidemiological characteristics of an outbreak of 2019 novel coronavirus diseases 
(COVID-19) in China. Zhonghua Liu Xing Bing Xue Za Zhi. 2020;41:145151.

5-Lu, H, Stratton, CW, Tang, YW. Outbreak of pneumonia of unknown etiology in Wuhan, China: the mystery and the miracle. J Med Virol. 2020;92:401-402.

6-Mahmudpour, M., Roozbeh, J., Keshavarz, M., Farrokhi, S., \& Nabipour, I. (2020). COVID-19 cytokine storm: The anger of inflammation. Cytokine, 133, 155151. https://doi.org/10.1016/j.cyto.2020.155151

1

7-Wu, Z, McGoogan, JM. Characteristics of and important lessons from the coronavirus disease 2019 (COVID-19) outbreak in China: summary of a report of 72314 cases from the Chinese Center for Disease Control and Prevention. JAMA. 2020;323:1239-1242.

8-Mosaddeghi, P, Negahdaripour, M, Dehghani, Z, et al. Therapeutic approaches for COVID-19 based on the dynamics of interferon-mediated .immune responses [published online ahead of print February 25, 2021]. Curr Signal Transd T. doi:10.2174/1574362416666210120104636.

9-Guan, W-j, Ni, ZY, Hu, Y, et al. Clinical characteristics of coronavirus disease 2019 in China. N Engl J Med. 2020;382:1708-1720. 'Google Scholar | Crossref | Medline

10-Zumla, A, Hui, DS, Azhar, El, Memish, ZA, Maeurer, M. Reducing mortality from 2019-nCoV: host-directed therapies should be an option. !Lancet. 2020;395:e35-e36.

11-Ulhaq, ZS, Soraya, GV. Interleukin-6 as a potential biomarker of ICOVID-19 progression. Med Mal Infect. 2020;50:382-383. 
12-Grifoni, A, Weiskopf, D, Ramirez, SI, et al. Targets of T cell responses to SARS-CoV-2 coronavirus in humans with COVID-19 disease and unexposed individuals. Cell. 2020;181:1489-1501.

13-Atal, S, Fatima, Z, Balakrishnan, S. Approval of itolizumab for COVID19: a premature decision or need of the hour? BioDrugs. 2020;34:705711.

14- Yendapally R, Sikazwe D, Kim SS, Ramsinghani S, Fraser-Spears R, Witte AP, La-Viola B. A review of phenformin, metformin, and imeglimin. Drug Dev Res. 2020 Jun;81(4):390-401. doi: 10.1002/ddr.21636. Epub 2020 Jan 9. PMID: 31916629.

15-Lehrer, Steven. "Inhaled biguanides and mTOR inhibition for influenza and coronavirus (Review)." World Academy of Sciences journal vol. 2,3 (2020): 1. doi:10.3892/wasj.2020.42

16-Kuratko, C.N. and Salem, N., Jr. (2013), Docosahexaenoic acid from algal oil. 'Eur. J. Lipid Sci. Technol., 115: 965-976. https://doi.org/10.1002/ejlt.20130006

17-Horrocks LA, Yeo YK. Health benefits of docosahexaenoic acid (DHA). Pharmacol Res. 1999 Sep;40(3):211-25. doi: 10.1006/phrs.1999.0495. PMID: 10479465.

18-Mani, NS, Budak, JZ, Lan, KF, et al. Prevalence of Coronavirus Disease 2019 infection and outcomes among symptomatic healthcare workers in Seattle, Washington. Clin Infect Dis. 2020;71:2702-2707.

19-Chakraborty, C, Sharma, AR, Bhattacharya, M, et al. COVID-19: consider IL-6 receptor antagonist for the therapy of cytokine storm syndrome in SARS-CoV-2 infected patients. J Med Virol. 2020;92:2260-2262. 
20-Cheng, F, Li, W, Zhou, Y, et al. admetSAR: a comprehensive source and free tool for assessment of chemical ADMET properties. J. Chem. Inf. Model. 2012;52:30993105.

21-Mills, N. ChemDraw Ultra 10.0 CambridgeSoft, 100 CambridgePark Drive, Cambridge, MA 02140. www.cambridgesoft.com. Commercial Price: 1910fordownload, 2150 for CD-ROM; Academic Price: 710fordownload, 800 for CDROM. J Am Chem Soc 2006;128:13649-13650.

22-Wang, J, Qiao, C, Xiao, H, et al. Structure-based virtual screening and characterization of a novel IL-6 antagonistic compound from synthetic compound database. Drug Des Devel Ther. 2016;10:4091-4100

23-Heo, L, Shin, WH, Lee, MS, Seok, C. GalaxySite: ligand-binding-site prediction by using molecular docking. Nucleic Acids Res. 2014;42:W210-W214.

24-Trott, O, Olson, AJ. AutoDock Vina: improving the speed and accuracy of docking with a new scoring function, efficient optimization, and multithreading. J Comput Chem. 2010;31:455-461.

25-Genheden, S, Ryde, U. The MM/PBSA and MM/GBSA methods to estimate ligand-binding affinities. Expert Opin Drug Discov. 2015;10:449-461. 